\title{
Target Object Tracking with Portable Sensors in Wireless Sensor Network
}

\author{
Musheer Vaquar, Sanjay Kumar Agarwal
}

\begin{abstract}
Detection and tracking of moving target objects is one of the important problems of wireless sensor systems. In recent years, portability has become an important research area for the WSN community. Although it was never thought that the WSN arrangement was completely stable, portability was initially perceived as some of the difficulties that must be overcome, including network, inclusion and use of vitality. Target object dictates the accuracy of target objects with which the position of target objects can be estimated. This problem becomes a test, especially given the portability of the sensor and the target object, in which the directions of the sensor and the target object have to be captured. [1, 17] Through this review, we consider that we consider the question of following signals that release appropriate target object using compact sensors that depend on the social issue of the signal. Since the versatile movement of the lens is unclear, the portable sensor controller uses the approximation accumulated by a remote sensor so that the compact article indicates the time of appearance (TOA). [4] The portable sensor controller confirms the TOA estimation information of both compact target object and portable sensors to evaluate their areas before guiding the development of portable sensors to achieve the target object. We propose the calculation of approximation (min-max) to calculate the monitoring area, which can be effectively understood through quasi-different programming (SDP), and apply a cubic potential to the portable sensor path Can do. We measure the area of portable sensors and focus on each other to improve the following accuracy.[2, 5] We determine the characteristic relationship between several basic parameters of the frame and the trackingtarget object, including the thickness of the sensor, the range of detection, the portability of the sensor, and the target object. We examine the relationships and the ability to influence by multiple parameters of the framework and locate the base number of portable sensors that are required to maintain the tracking of target objects in an MSN. To further improve the execution of the framework, we propose a weighted monitoring calculation, using estimation information more efficiently.[3] Our entertainment results suggest that insufficiently insured calculations provide excellent follow-up that can be improved by requesting greatness with an equal number of sensors when contrasted and with the position of static sensors.
\end{abstract}

Keywords: Wireless Sensor Networks, Portable sensor, Target Object Tracking, Static Sensor

\section{INTRODUCTION}

The advancement of sensor network technology has allowed the target location to be monitored and monitored in dense conditions.

Revised Manuscript Received on December 30, 2019.

* Correspondence Author

Musheer Vaquar*, Research Scholar, Sri Venkateswara University Gajraula, Uttar Pradesh-India

Prof.(Dr.) Sanjay Kumar Agarwal, Professor, Dolphin (PG) Institute of Biomedical \& Natural Sciences, Dehradun, Uttarakhand-India

(C) The Authors. Published by Blue Eyes Intelligence Engineering and Sciences Publication (BEIESP). This is an open access article under the CC BY-NC-ND license (http://creativecommons.org/licenses/by-nc-nd/4.0/)
$[8,15]$ The enthusiasm for the organization of portable sensors for target tracking has been enhanced, driven primarily by the interest of critical location monitoring and wildlife (illegal tracking of rare wild animals), robotic navigation, search-rescue, and autonomous surveillance [1, 7]. For the most part, goal tracking involves two steps:

1) It must measure or anticipate the target area from the noise information evaluated by the sensor.

2) Itneeds to control a portable sensor_tracker to chase or catch a moving_ target.

Through this research paper, we are primarily motivated to study the topic of portable target area in a sensor organization consisting of fixed sensors and a portable sensor. In particular, we are interested in controlling the portable sensor to measure the target object position and track the mobility of target object.[4]

\section{Proposed Advanced Contribution:}

Through this work, we have a significant impetus to track target object articles on mobile targets and portable sensors that use the time of appearance model. In particular, we are interested in maintaining the shock of inference due to the propagation of many routes and the detection of errors. With the foundations of the TOA model, we propose an estimation approach (minimum-maximum) to assess the situation that can be solved in a simple and effective way through techniques for the lack of semi-defined programming (SDP). We do. We apply cubic capability to investigate the practice of compact sensors.[5,6] In the same way, we also checks the range of portable sensors and target article to further improve the accuracy of tracking. We present a continuous weighted monitoring method to use more information than approximation. The measurable result speaks of the fact that the following proposed calculations work exceptionally well. There are some important explanations behind us for using the TOA estimation model. In any case, performing an approximation of the TOA is easy, because each sensor simply needs to recognize a single sine component, for example, to record its presence time in order to record its presence. Second, our particular use of TOA is an increasingly practical model, as we do not need to mess with the sensor to understand the transmission time of the previous signal.[18, 19] In this sense, our TOA model allows us to coordinate the evaluation of the original situation by configuring TOA estimation information. The paper is organized as the following segment. In the next segment, we call the following routing problem that defines a compact target and controls a portable sensor.[14, 16] In the final field our measurable results are seen and then we complete the work. 


\section{Target Object Tracking with Portable Sensors in Wireless Sensor Network}

\section{PORTABLE SENSOR GUIDE STRATEGY}

A guide asked to control the portable sensor to attract a moving target object from any basic position in this position. Since the movements of target objective elements are not recognized before the controller, a continuous process is required to deal with the problem.

We estimate the time of appearance at the anchor node $x_{i}$ at times for individual target $T_{j}$ markings and portable sensors,

$$
\begin{aligned}
& t_{j i}=\frac{1}{c}\left\|x_{i}-y_{j}\right\|+t_{j 0}+\frac{1}{c}\left\|x_{i}-y_{j}\right\| n_{j i}+\delta_{j}(\mathrm{~A}) \\
& \mathcal{T}_{j i}=\frac{1}{c}\left\|x_{i}-z_{j}\right\|+\mathcal{T}_{j 0}+\frac{1}{c}\left\|x_{i}-z_{j}\right\| \eta_{j i}+\rho_{j}(\mathrm{~B})
\end{aligned}
$$

Here, $c$ is the speed of the traveling signal, $t_{j 0}, \mathcal{T}_{j 0}$ are respectively, moments in time when the target and portable sensors transmit their signal. Also note that with $\frac{1}{c} \| x_{i}-$ $y_{j}\left\|n_{j i}, \frac{1}{c}\right\| x_{i}-z_{j} \| \eta_{j i}$ with $n_{j i} \geq 0, \eta_{j i} \geq 0$ is a multi-path engraved noise, while $\delta_{j}$ and $\rho_{j}$ are a setback to detect errors.

Furthermore, we have an estimate of the presence time on the portable sensor at the time of $T_{j}$ for the target signal $\varphi_{j}=\frac{1}{c}\left\|y_{j}-z_{j}\right\|+t_{j 0}+\frac{1}{c}\left\|y_{j}-z_{j}\right\| \zeta_{j}+k_{j}(\mathrm{C})$ Where $\frac{1}{c}\left\|y_{j}-z_{j}\right\| \zeta_{j}$ with $\zeta_{j} \geq 0$ represents the multipath propagation noise, and $k_{j}$ is the noise due to sensing error.

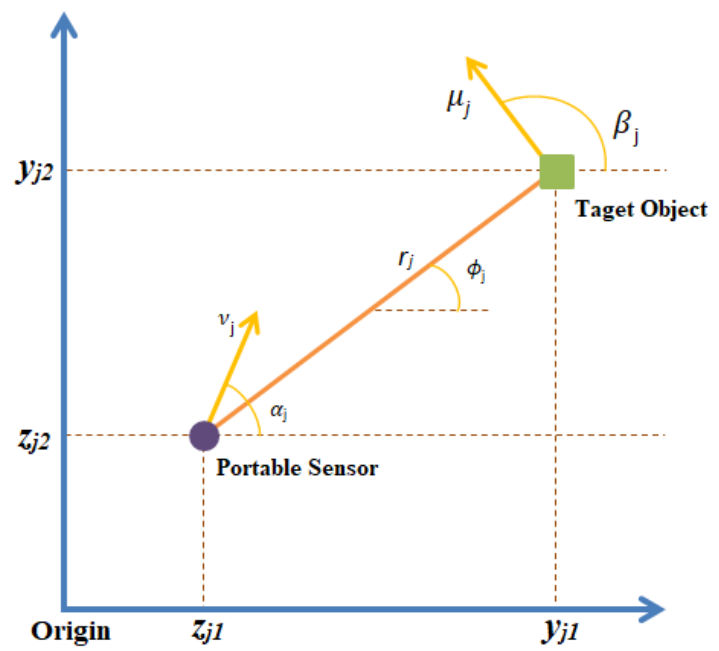

Figure_1: Graphic of Navigation Problem Analysis In the figutr_1, we are show the geometric model of the course subject in 2-dimension plane. At the time of $T_{j}$, the portable sensor is arranged $z_{j}=\left[z_{j 1}, z_{j 2}\right]^{T}$ twith a velocity rate $v_{j}$ and the edge $\alpha_{j}$ into the main quadrant of the plane and the target element located at $y_{j}=\left[y_{j 1}, y_{j 2}\right]^{T}$ with a velocity rate $u_{j}$ and the point of angle $\beta_{j}$ for a uniform square. The extended line that displays the portable sensor and target element with $r_{j}$ is the main quadrant with angle $\phi_{j}$ to the point quadrate. Then, the portable sensor and kinematics of the target object is as follows;

$\dot{z_{j 1}}=v_{j} \cos \alpha_{j}, \dot{z_{j}}=v_{j} \sin \alpha_{j}$

$\dot{y_{j 1}}=u_{j} \cos \beta_{j}, \dot{y_{j 2}}=u_{j} \sin \beta_{j}$

Since $\tan \phi_{j}=\frac{y_{j 2}-z_{j 2}}{y_{j 1}-z_{j 1}}$, the decomposition of the relative velocity gives the relative kinematic position equation that accompanies it between the portable sensor and the target object.

$\dot{r}_{j}=\mu_{j} \cos \left(\beta_{j}-\phi_{j}\right)-v_{j} \cos \left(\alpha_{j}-\phi_{j}\right)$

$r_{j} \dot{\phi}_{j}=\mu_{j} \sin \left(\beta_{j}-\phi_{j}\right)-v_{j} \sin \left(\alpha_{j}-\phi_{j}\right)$
In the event that $\dot{r}_{J}<0$, at that point the separation between the portable sensor and the target object is diminishing, i.e., the portable sensor is moving toward the target object. As indicated by explore [13], a cubic route navigation technique has been proposed, where

$\alpha_{j}=\phi_{j}+K \phi_{j}^{3}$

By accepting $v_{j}>\mu_{j}$, it is shown that under this cubic route law, the comparison $\dot{r}_{j}<0$, and the portable sensor will effectively reach the target. In light of the simplicity of this route navigation law, we will apply this method in our research work.

On the other hand, we may be interested in keeping portable sensors separate with purposefully manners without causing detection reasons. In such applications, we have to integrate $\dot{r}_{j}=0$. Merging equation $(\mathrm{F})$ and $(\mathrm{H})$, we have

$\mu_{j} \cos \left(\beta_{j}-\phi_{j}\right)=v_{j} \cos \left(K \phi_{j}^{3}\right) \quad$ (I)

which gives the portable sensor speed as $v_{j}=\frac{\mu_{j} \cos \left(\beta_{j}-\phi_{j}\right)}{\cos \left(K \phi_{j}^{3}\right)}(\mathrm{J})$

\section{TARGET OBJECTS TRACKING ALGORITHM}

This algorithm have several steps are as follows;

\section{A. Target Localization}

The initial step to perform the target object tracking is to measure actual position of the target object and of portable sensors. Since the TOA data collected in the information combination focus is the same for both target object and portable sensors, therefore, along these lines, we focus on how to measure the area vector $v_{j}$ and the target object of $T_{j}$. We can adjust the TOA model by renewing (A)

$t_{j i}-t_{j 0}=\frac{1}{c}\left\|x_{i}-y_{j}\right\|+\frac{1}{c}\left\|x_{i}-y_{j}\right\| n_{j i}+\delta_{j}(\mathrm{~K})$

By squaring both sides we get

$\left(t_{j i}-t_{j 0}\right)^{2}-\frac{1}{c^{2}}\left\|x_{i}-y_{j}\right\|^{2}=\left(\frac{1}{c}\left\|x_{i}-y_{j}\right\| n_{j i}+\right.$

$\left.\delta_{j}\right)\left(\frac{1}{c}\left\|x_{i}-y_{j}\right\|\left(2+n_{j i}\right)+\delta_{j}\right)$

fori $=1,2,3 \ldots \ldots .$.

Right side of the equation (L) indicates the noise term $\omega_{j i}$ which is sovereign for many different indices $i$. In the event that $n_{i j}$ and $\delta_{j}$ are zero, then to the right side of equation (L) will be zero. In this sense, one approach to measuring the ideal $y_{j}$ without accepting specific characteristics in $\omega_{j i}$ is to limit the standard $\ell_{\infty}$ of $\omega_{j i}$. This methodology does not make assumptions about the transport of uproar or belief in uproar. Just try to limit peak error. Next, it is necessary that your presentation be less delicate with an appropriation or ruckus relationship.[12] In this sense, we propose to obtain a minimum-maximum (minmax) basis for the estimation of this region.

$\hat{y}_{j}=\arg \max _{y_{j}} \max _{i=1,2, \ldots N}\left|\left(t_{j i}-t_{j 0}\right)^{2}-\frac{1}{c^{2}}\left\|x_{i}-y_{j}\right\|^{2}\right|$

(M)

The min-max equation $(M)$ is non-convex, but is relativelyresponsive to semi-definite relaxations as shown below. Initially we introduce two auxiliary variables $y_{j s}=$ $\boldsymbol{y}_{j}^{T} \boldsymbol{y}_{j}$ and $t_{j s}=t_{j 0} . t_{j 0}$, and define the following function:

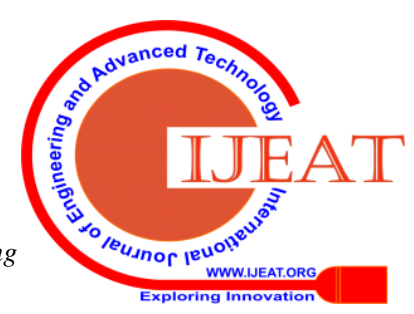


$\psi\left(t_{j s}, t_{j i}, t_{j 0}, y_{j s}, x_{i}, y_{j}\right)=2 t_{j i}^{2}-t_{j i}^{2}-\frac{1}{c^{2}}\left(y_{j s}-2 x_{i}^{T} \boldsymbol{y}_{j}+\right.$ $\left.x_{i}^{T} x_{i}\right)$

The equation (M) can be rewrite as;

$\hat{y}_{j}=\arg \min _{y, y_{j s}, t_{j 0}, t_{j s,}, i=1,2, \ldots . . N}\left|\psi\left(t_{j s}, t_{j i}, t_{j 0}, y_{j s}, x_{i}, y_{j}\right)\right|$

which is a convex function of $y_{j}, y_{j s}, t_{j 0}$ and $t_{j s}$.

Be that as it may, the two equalities $y_{j s}=\boldsymbol{y}_{j}^{T} \boldsymbol{y}_{j}$ and $t_{j s}=$ $t_{j 0} \cdot t_{j 0}$ are not relatives. To arc the entire definition, we loosen the two correspondences $y_{j s}=\boldsymbol{y}_{j}^{T} \boldsymbol{y}_{j}$ and $t_{j s}=t_{j 0} \cdot t_{j 0}$ with inequalities $y_{j s} \geqslant \boldsymbol{y}_{j}^{T} \boldsymbol{y}_{j}$ and $t_{j s} \geqslant t_{j 0} . t_{j 0}$, individually. These imbalances can also be communicated in direct matrix inequalities, i.e.

$$
\left[\begin{array}{cc}
\mathbf{I} & \boldsymbol{y}_{j} \\
\boldsymbol{y}_{j}^{T} & \boldsymbol{y}_{s}
\end{array}\right] \geqslant 0,\left[\begin{array}{cc}
1 & t_{j 0} \\
t_{j 0} & t_{j s}
\end{array}\right] \geqslant 0
$$

Furthermore, given the time of the estimate position $T_{j-1}$, we can obtain the vector of the area assumed for the target at time $T_{j}$. Let $\triangle T_{j}=T_{j}-T_{j-1}$ and $\mu_{j-1}$ are the velocity vectors determined for the target $T_{j-1}$. At that point, the change in area can be approximated as $\Delta \boldsymbol{y}_{j}=y_{j}-y_{j-1} \approx$ $\triangle T_{j} \mu_{j-1}$. This can be used as an additional imperative for estimating the target area in $T_{j}$ time. Considering 2D, the field transformation vector into $\Delta y_{j}$ is limited to one case, at which point the comparison $y_{j}$ will also be forced into a box, i.e.,

$$
y_{j l} \leq y_{j 1} \leq y_{j r}, y_{j d} \leq y_{j 2} \leq y_{j u}
$$

Define $a_{j}=\left[y_{j l} y_{j d}\right]^{T}, \quad b_{j}=\left[y_{j r} y_{j u}\right]^{T}$ and $y_{j s}=y_{j}^{T} y_{j}$. We can apply the Reformulation-Linearization Technique (RLT) [16] to (Q) to obtain some additional limitations. Indeed, in view of the RLT (Q) it may be loose as;

$$
\left.\begin{array}{c}
a_{j}^{T} a_{j}-a_{j}^{T} y_{j}-a_{j}^{T} y_{j}+y_{j s} \geq 0 \\
b_{j}^{T} b_{j}-b_{j}^{T} y_{j}-b_{j}^{T} y_{j}+y_{j s} \geq 0 \\
-a_{j}^{T} b_{j}+a_{j}^{T} y_{j}+b_{j}^{T} y_{j}-y_{j s} \geq 0
\end{array}\right\}
$$

which can be rewritten in the following matrix form

$$
\left[\begin{array}{ccc}
\left\|a_{j}\right\|^{2} & -2 a_{j}^{T} & 1 \\
\left\|b_{j}\right\|^{2} & -2 b_{j}^{T} & 1 \\
-a_{j}^{T} b_{j} & a_{j}^{T}+b_{j}^{T} & -1
\end{array}\right]\left[\begin{array}{c}
1 \\
y_{j} \\
y_{j s}
\end{array}\right] \geq 0
$$

Here " $\geq 0$ " means that every component in the vector is not negative. By consolidating the above requirements, we derive the definition of SDP rationalization that accompanies:

$$
\begin{gathered}
\min _{y_{j}, y_{j s}, t_{j 0}, t_{j s}} \theta_{j} \\
{\left[\begin{array}{cc}
\mathbf{I} & y_{j} \\
y_{j}^{T} & y_{j s}
\end{array}\right] \geqslant 0,\left[\begin{array}{ccc}
\mathbf{I} & t_{j 0} \\
t_{j 0} & t_{j s}
\end{array}\right] \geqslant 0} \\
{\left[\begin{array}{ccc}
\left\|a_{j}\right\|^{2} & -2 a_{j}^{T} & 1 \\
\left\|b_{j}\right\|^{2} & -2 b_{j}^{T} & 1 \\
-a_{j}^{T} b_{j} & a_{j}^{T}+b_{j}^{T} & -1
\end{array}\right]\left[\begin{array}{c}
1 \\
y_{j} \\
y_{j s}
\end{array}\right] \geq 0}
\end{gathered}
$$

This problem $\mathrm{T}$ can be solved using common mean.

\section{B. Portable Sensor Localization}

Along with evaluating the position of the target object, we can rectify the problem of limiting compact sensors to the problem of not devising SDPs. More explicitly, we can evaluate the compact area of the $z_{j}$ sensor through a comparative definition that relies on the TOA estimates at

the suspension centres of the sine derived from the portable sensor (B).

Define $z_{j s}=\boldsymbol{z}_{j}^{T} \boldsymbol{z}_{j}$ and $\mathcal{T}_{j s}=\mathcal{T}_{j 0}$. $\mathcal{T}_{j 0}$. Similarly, on the bases of given velocity-vector $v_{j-1}$ of the portable- sensor from the controllers at time instant $T_{j-1}$, we can approximate the location change of the portable sensor as $\triangle z_{j}=z_{j}-z_{j-1} \approx$ $\triangle T_{j} v_{j-1}$. The corresponding $z_{j}$ will be the constraint to a box, i.e.

$$
z_{j l} \leq z_{j 1} \leq z_{j r}, z_{j d} \leq z_{j 2} \leq z_{j u}
$$

Let $d_{j}=\left[z_{j l} z_{j d}\right]^{T}$ and $e_{j}=\left[z_{j r} z_{j u}\right]^{T}$. By applying the similar relaxations, we obtain the following SDP formulation:

$$
\begin{gathered}
\min _{z_{j,}, z_{j s}, T_{j 0}, T_{j s}} \theta_{j} \\
\text { s.t. }-\theta_{j}<\psi\left(\mathcal{T}_{j s}, \mathcal{T}_{j i}, \mathcal{T}_{j 0}, z_{j s}, z_{i}, z_{j}\right)<\theta_{j} \\
{\left[\begin{array}{cr}
\mathbf{I} & z_{j} \\
z_{j}^{T} & z_{j s}
\end{array}\right] \geqslant 0,\left[\begin{array}{cc}
\mathbf{I} & \mathcal{T}_{j 0} \\
\mathcal{J}_{j 0} & \mathcal{T}_{j s} a
\end{array}\right] \geqslant 0 \quad(\mathrm{~V})} \\
{\left[\begin{array}{ccc}
\left\|d_{j}\right\|^{2} & -2 e_{j}^{T} & 1 \\
\left\|e_{j}\right\|^{2} & -2 e_{j}^{T} & 1 \\
-d_{j}^{T} e_{j} & d_{j}^{T}+e_{j}^{T} & -1
\end{array}\right]\left[\begin{array}{c}
1 \\
z_{j} \\
z_{j s}
\end{array}\right] \geq 0}
\end{gathered}
$$

\section{Combined Target Portable Sensor_Localization}

The compact sensor node received target-signal data and may receive additional TOA estimation from the target to the portable-sensor (C). This gives a link between the target and compact regions of the TOA data sensor. If in the case $\boldsymbol{z}_{j}$ is known as (C), we can consider the compact sensor as another center of stability and include a greater inequality of (T). This additional data can be obtained using the $\boldsymbol{z}_{j}$ display in $(\mathrm{V})$ and then $(\mathrm{C})$. In any case, since we are not ready to acquire the exact $\boldsymbol{z}_{j}$, it will generate a generated error. In this way, we propose to understand both $\boldsymbol{y}_{j}$ and $\boldsymbol{z}_{j}$ at all times to make more use of (C) to TOA estimation data.

In order to achieve this we required extra variable to convert into convex. Let $q_{j}=y_{j}^{T} z_{j}$, then $\left\|y_{j}-z_{j}\right\|^{2}=y_{j s}-2 q_{j}+$ $z_{j s}$. And we have the following constraint:

$$
-\frac{1}{2}\left(y_{j s}+z_{j s}\right) \leq q_{j} \leq \frac{1}{2}\left(y_{j s}+z_{j s}\right)(\mathrm{W})
$$

Merging the above formulation and constraints, we get at the joint optimization-formulation as:

$$
\begin{gathered}
\min _{y_{j}, y_{j s}, t_{j 0}, t_{j s}, z_{j}, z_{j s}, T_{j 0}, T_{j s}, q_{j}} \theta_{j} \\
\text { s.t. }-\theta_{j}<\psi\left(t_{j s}, t_{j i}, t_{j 0}, y_{j s}, x_{i}, y_{j}\right)<\theta_{j} \\
-\theta_{j}<\psi\left(\mathcal{T}_{j s}, \mathcal{J}_{j i}, \mathcal{T}_{j 0}, z_{j s}, x_{i}, z_{j}\right)<\theta_{j}, \\
-\theta_{j}<-t_{j s}-\varphi_{j} t_{j 0}+\varphi_{j}^{2}-\frac{1}{c^{2}}\left(y_{j s}-2 q_{j}+z_{j s}\right)<\theta_{j}, \\
{\left[\begin{array}{cc}
\mathbf{I} & y_{j} \\
y_{j}^{T} & y_{j s}
\end{array}\right] \geqslant 0,\left[\begin{array}{cc}
\mathbf{I} & t_{j 0} \\
t_{j 0} & t_{j s}
\end{array}\right] \geqslant 0}
\end{gathered}
$$$$
\left[\begin{array}{lcc}
\left\|a_{j}\right\|^{2} & -2 a_{j}^{T} & 1 \\
\left\|b_{j}\right\|^{2} & -2 b_{j}^{T} & 1 \\
-a_{j}^{T} b_{j} & a_{j}^{T}+b_{j}^{T} & -1
\end{array}\right]\left[\begin{array}{c}
1 \\
y_{j} \\
y_{s}
\end{array}\right] \geq 0
$$$$
-\frac{1}{2}\left(y_{j s}+z_{j s}\right) \leq q_{j} \leq \frac{1}{2}\left(y_{j s}+z_{j s}\right),
$$

$$
\begin{gathered}
{\left[\begin{array}{cc}
\mathbf{I} & z_{j} \\
z_{j}^{T} & z_{j s}
\end{array}\right] \geqslant 0,\left[\begin{array}{cc}
\mathbf{1} & \mathcal{T}_{j 0} \\
\mathcal{T}_{j 0} & \mathcal{T}_{j s}
\end{array}\right] \geqslant 0} \\
{\left[\begin{array}{lcc}
\left\|d_{j}\right\|^{2} & -2 e_{j}^{T} & 1 \\
\left\|e_{j}\right\|^{2} & -2 e_{j}^{T} & 1 \\
-d_{j}^{T} e_{j} & e_{j}^{T}+e_{j}^{T} & -1
\end{array}\right]\left[\begin{array}{c}
1 \\
z_{j} \\
z_{j s}
\end{array}\right]}
\end{gathered}
$$




\section{Target Object Tracking with Portable Sensors in Wireless Sensor Network}

More generally, multiple portable sensors can be deployed and multiple TOA estimation can be utilized. Extending the single-portable sensor formulation of $(\mathrm{X})$, we have multiple $z_{j}^{\prime} s$ to estimate. Without displaying the formulation in detail, we can see that it is clear to generalize the formation (X) in order to consider multiple measurements of multipleportable sensors.

\section{Conditions for Localization}

We identified that the source constraint is not unlimited and on the bases geography of the sensor. As it appeared [11, 18], in the 2-dimensionplane, if all the attached sensor centres are in a secluded line, that is, they collide with each other, then the problem of the region of origin becomes poorly adapted at that time and the results are revealed. To tell the confusing truth, there may be several emerging areas when no additional data are provided beyond the TOA estimates.[9, 10] Generally, we may occasionally experience collier conditions while tracking the target object. As it may be, given that we have other previous data on the area of instantaneous(s) of target object (Q) as well as its multifaceted motion, this previous information gives us the field disturbances caused by the collinear sensor. Positively, we really include these data in priority in our scheme (X). In this sense, the ambiguity in our response (X) can be resolved.

\section{SIMULATION ENVIRONMENT AND RESULTS:}

In our investigation, we considered arbitrarily moving a system of 5portablesensors over a field region. The portable sensor controller is located at the highest point in the field. The radio transmission range of the sensor nodes is 20 meters. The sensor centres move irregularly with an uncontrolled estimate of speed in the range 1-4 m / s.

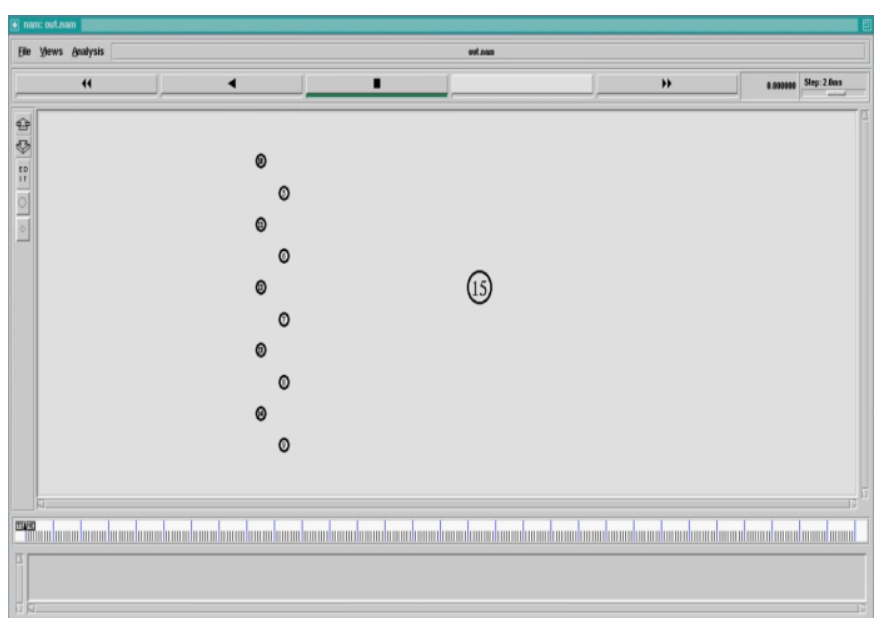

Figure_2: Simulation setup initial state

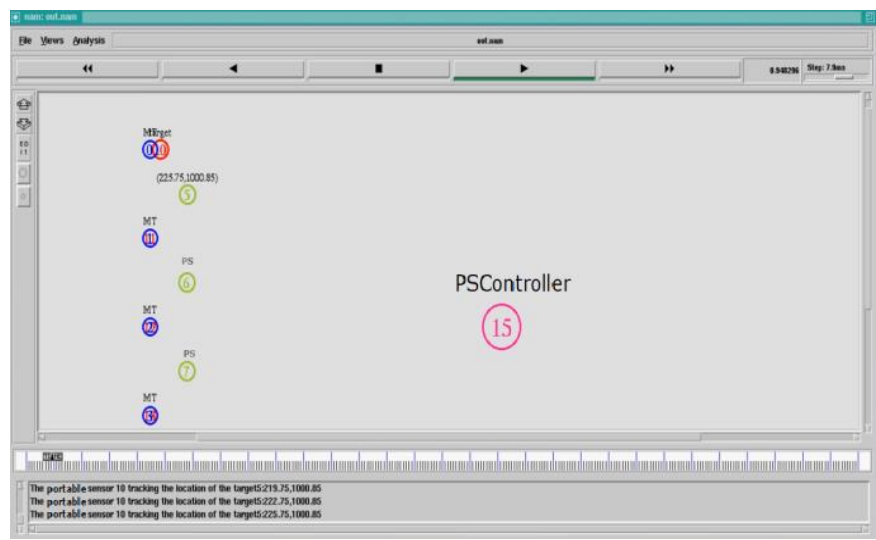

Figure_3: Portable Sensor (PS)ready to sense target object number 10

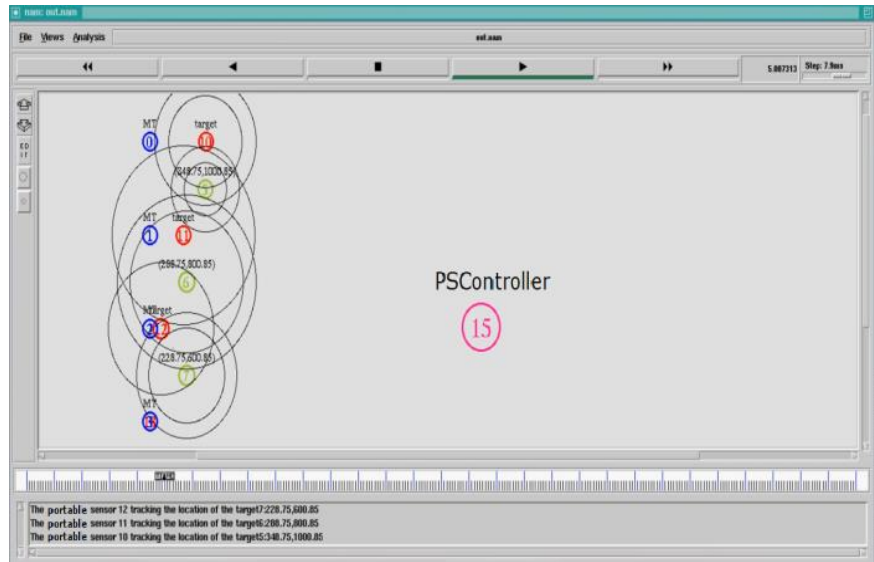

Figure_4: Portable Sensors (PSs)activated and start sensing target object number 10,11 and 12

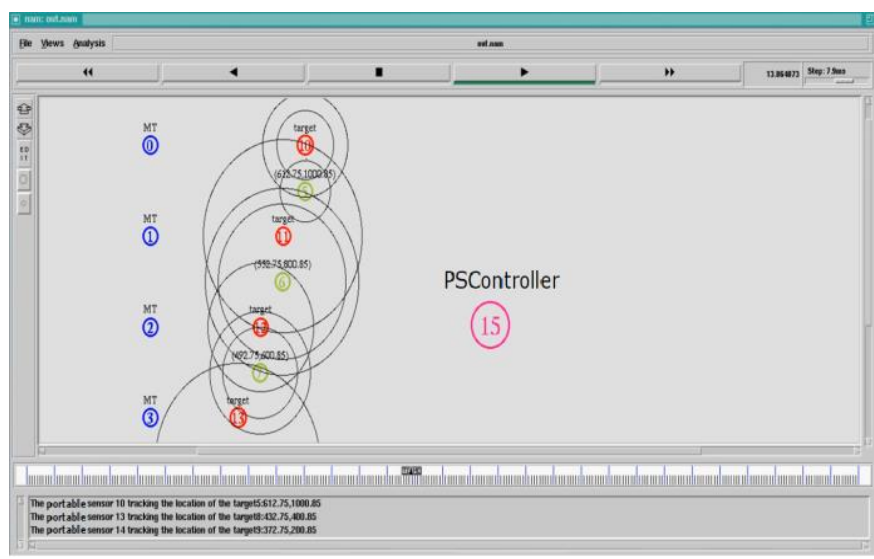

Figure_5: Portable Sensors (PSs) sense and track the target object number 


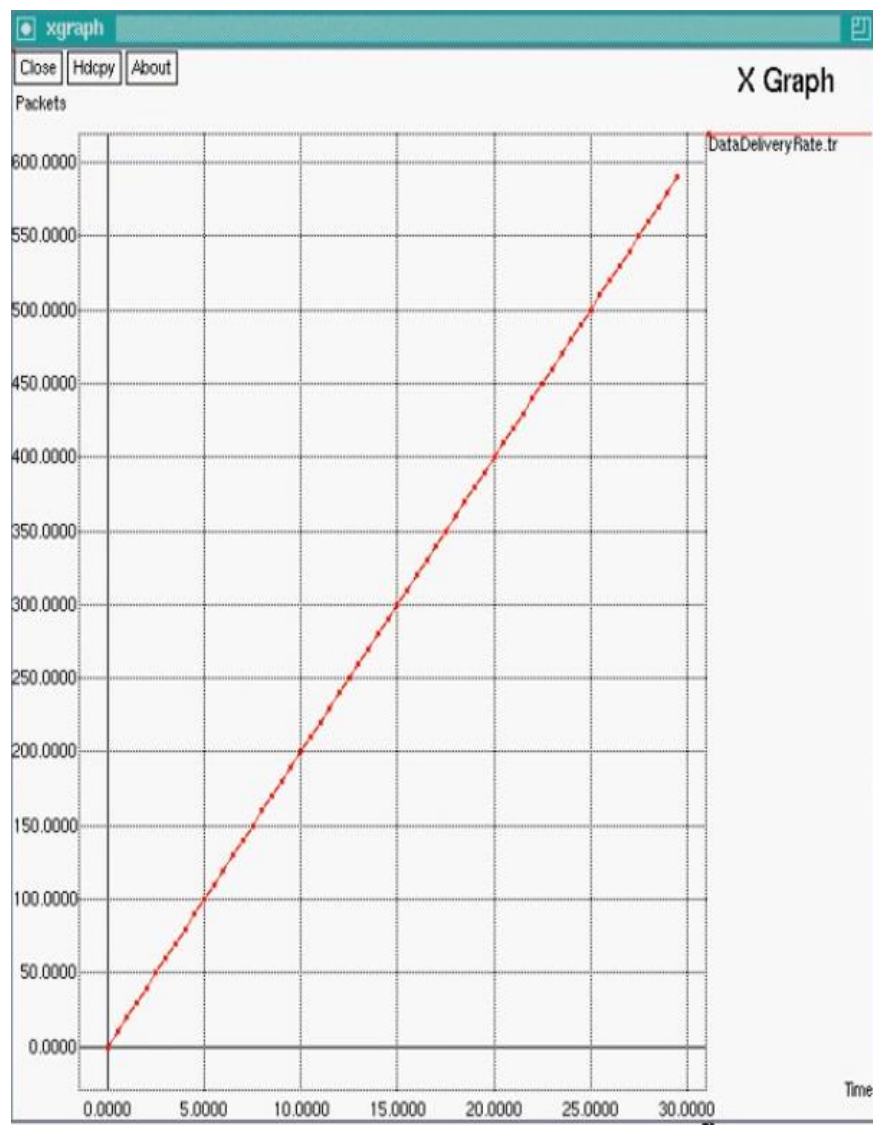

Figure_6: Data Delivery Rate over the portable sensor and the target object

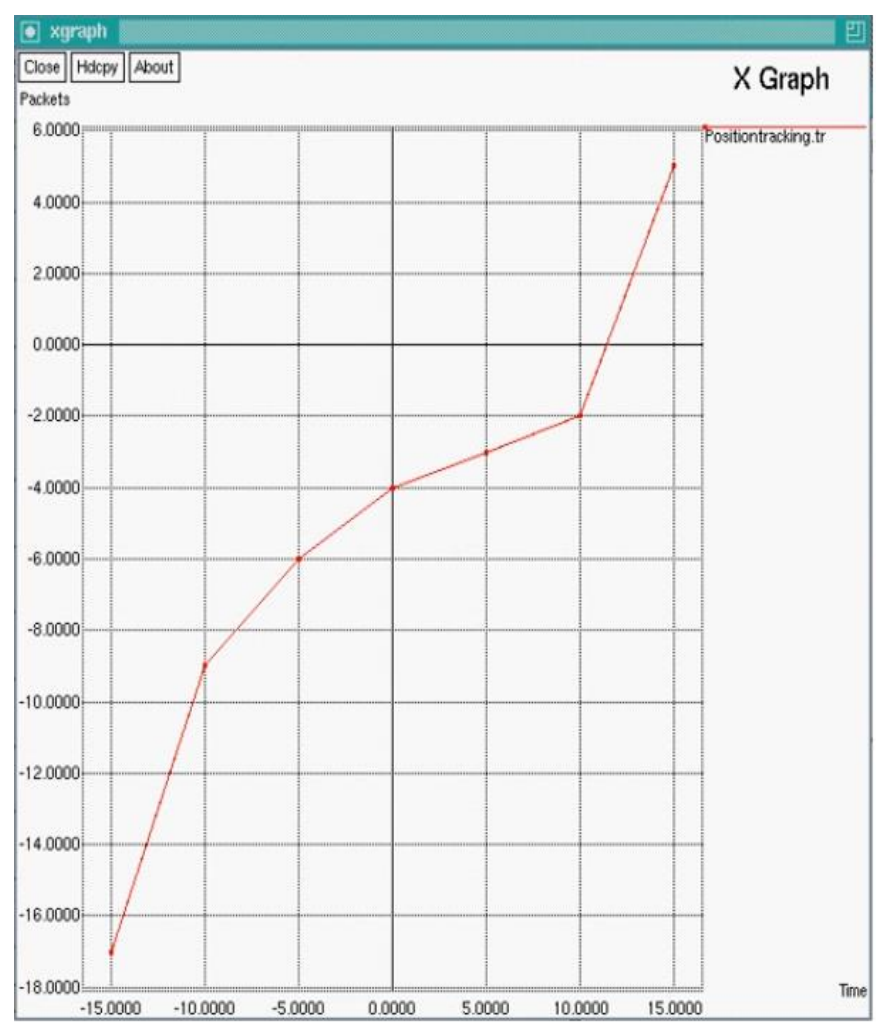

Figure_7: Target object position tracking graph

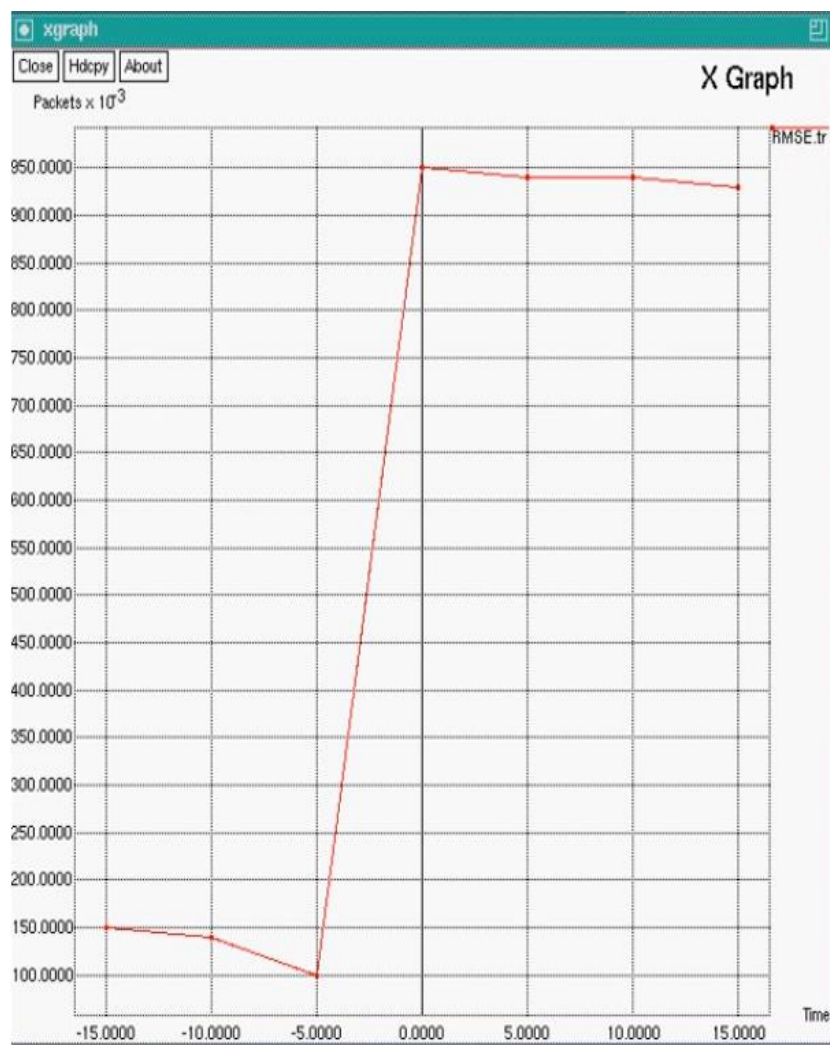

Figure_8: Root_Mean_Squared_Error graph

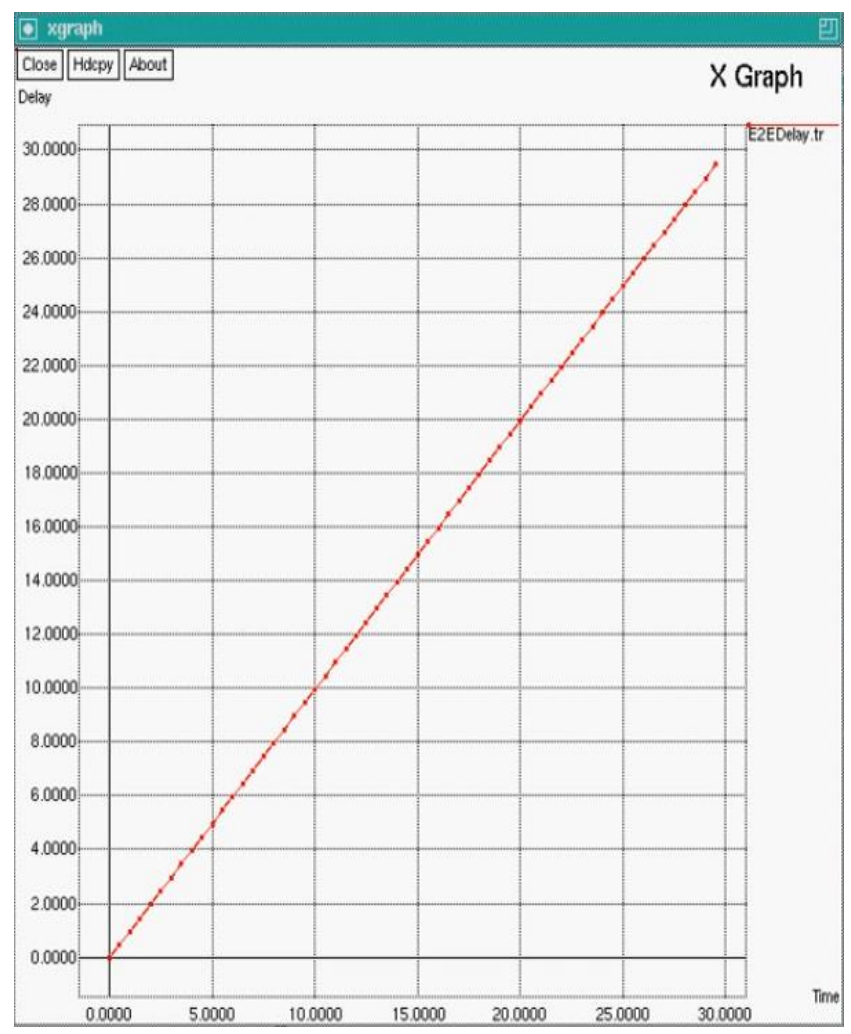

Figure_9: Latency graph 


\section{Target Object Tracking with Portable Sensors in Wireless Sensor Network}

\section{CONCLUSION}

In this article, we have considered the target tracking problem in wireless sensor systems. In particular, we present performance measures: examining portability of sensor against spatial target object and target object mobility. Showing the dynamic parts of target object tracking that depend on the portability of both the sensor node and the target object, we infer the expected relationship between spatial target objects and several key parameters of the frame, including the target object detection range of the sensor, density of the sensors, sensor nature and target object mobility. The outcomes showed that portability can be broken to achieve better spatial object resolution. Some ways to do more research on this topic are: (A) Consider the recognition error of portable sensors on the speed of a fluctuating sensor. This may broaden into an improvement problem for tracking purposes; (B) Correspondence between the sensor versatility model, the system model, and the sensor model to allow sophisticated detection and tracking of the model. For example, a scattered distributedtarget object that follows and traces the data trade convention becomes an attractive subject of future research when the sensor is required to follow the target object route.

\section{REFERENCES:}

1. Cetin, M., Chen, L., Fisher, J. W., Ihler, A. T., Moses, R. L., Wainwright, M. J., \&Willsky, A. S. (2006). Distributed fusion in sensor networks. IEEE Signal Processing Magazine, 23(4), 42-55.

2. Sayed, A. H., Tarighat, A., \&Khajehnouri, N. (2005). Network-based wireless location: challenges faced in developing techniques for accurate wireless location information. IEEE signal processing magazine, 22(4), 24-40.

3. Patwari, N., Ash, J. N., Kyperountas, S., Hero, A. O., Moses, R. L., \&Correal, N. S. (2005). Locating the nodes: cooperative localization in wireless sensor networks. IEEE Signal processing magazine, 22(4), 5469.

4. Tseng, P. H., Feng, K. T., Lin, Y. C., \& Chen, C. L. (2009). Wireless location tracking algorithms for environments with insufficient signal sources. IEEE Transactions on Mobile Computing, 8(12), 1676-1689.

5. Li, T., Ekpenyong, A., \& Huang, Y. F. (2006). Source localization and tracking using distributed asynchronous sensors. IEEE Transactions on Signal Processing, 54(10), 3991-4003.

6. Mihaylova, L., Angelova, D., Bull, D., \&Canagarajah, N. (2010). Localization of mobile nodes in wireless networks with correlated in time measurement noise. IEEE Transactions on Mobile Computing, 10(1), 44-53.

7. Zou, Y., \&Chakrabarty, K. (2007). Distributed mobility management for target tracking in mobile sensor networks. IEEE Transactions on Mobile Computing, 6(8), 872-887.

8. Rao, R., \&Kesidis, G. (2004). Purposeful mobility for relaying and surveillance in mobile ad hoc sensor networks. IEEE Transactions on Mobile Computing, 3(3), 225-231.

9. Mehrandezh, M., Sela, M. N., Fenton, R. G., \&Benhabib, B. (2000). Proportional navigation guidance for robotic interception of moving objects. Journal of Robotic Systems, 17(6), 321-340.

10. Shames, I., Dasgupta, S., Fidan, B., \& Anderson, B. D. (2009, August). Circumnavigation using distance measurements. In 2009 European Control Conference (ECC) (pp. 2444-2449). IEEE.

11. Belkhouche, F., Belkhouche, B., \&Rastgoufard, P. (2006). Line of sight robot navigation toward a moving goal. IEEE Transactions on Systems, Man, and Cybernetics, Part B (Cybernetics), 36(2), 255-267.

12. Vargas, J., Mendez, S., \&Belkhouche, F. (2009, October). Tracking under the nonholonomic constraint using cubic navigation laws. In 2009 IEEE International Conference on Systems, Man and Cybernetics (pp. 2788-2793). IEEE.

13. Xu, E., Ding, Z., \&Dasgupta, S. (2011). Source localization in wireless sensor networks from signal time-of-arrival measurements. IEEE Transactions on Signal Processing, 59(6), 2887-2897.

14. Biswas, P., Liang, T. C., Toh, K. C., Ye, Y., \& Wang, T. C. (2006). Semidefinite programming approaches for sensor network localization with noisy distance measurements. IEEE transactions on automation science and engineering, 3(4), 360-371.

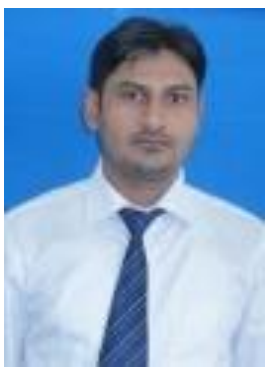

\section{AUTHORS PROFILE} various research papers in reputed journals and different national and international conferences.

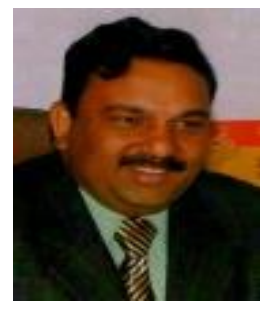

Prof.(Dr.) Sanjay Kumar Agarwal is in profession of teaching and research for the last twenty years to under graduate and post graduate student. He wrote six best seller books, published and presented 28 research papers in national and international journals. he is also associated with universities in the capacity of member of Board of studies and research development committee. Presently five research scholars are actively doing research with him on management, computer science and mathematics.
MusheerVaquar, Research Scholar, Sri Venkateswara University Gajraula, Uttar professor in Department of Computer Science and Engineering at Uttaranchal University, industry with 11 years of teaching an B.Tech degree from H.N.B Garhwal University, Shrinagar, M.Tech degree from Graphic Era University, Dehradun and now he is pursuing Ph.D. from Sri Venkateswara University Gajraula, Uttar Pradesh.

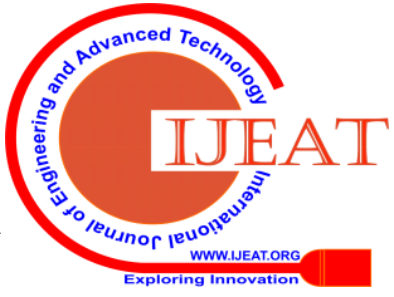

